\title{
Adaptive Fuzzy Type-2 Synergetic Control Based on Bat Optimization for Multi-Machine Power System Stabilizers
}

\author{
Emira Nechadi \\ Ferhat Abbas Setif 1 University \\ Setif, Algeria \\ emira.nechadi@univ-setif.dz
}

\begin{abstract}
A new, adaptive, fuzzy type-2 fast terminal, synergetic multi-machine power system stabilizer is proposed in this study, based on the Bat algorithm. The time spent to reach the equilibrium point, from any initial state, is guaranteed to be finite. The adaptive fuzzy type- 2 design is applied to estimate the unknown functions of a multi-machine power system. The parameters of the fast terminal synergetic control are optimized, using bat metaheuristic method. In order to test the robustness of the proposed stabilizer, three load conditions, of the multimachine power system are studied. A comparison of the proposed adaptive fuzzy type-2 synergetic power system stabilizer with bat conventional approach is presented, indicating improved performance. The control system stability is assessed by the second theorem of Lyapunov and is proven to be asymptotically stable.
\end{abstract}

Keywords-adaptive fuzzy type-2 design; fast terminal synergetic control; bat algorith m; Lyapunov stability; power system stabilizer

\section{INTRODUCTION}

A power system must remain stable and capable of withstanding a wide range of disturbances, in order to provide secure and reliable services. In a power system, the active power depends on the phase angle between the sending and receiving-end voltages, whereas the reactive power depends on the voltage magnitudes. A dynamic model of the system can be described, by the relationships between active and reactive powers and the bus voltage and frequency [1]. In a stable power system, when synchronous generators are subjected to a disturbance, they either return quickly to their original state or to a new stable operating point. Disturbances cause mechanical oscillations, which must be damped [2]. Power systems are complex nonlinear systems that often exhibit low frequency oscillations, due to insufficient damping caused by adverse operating conditions, which can lead the underlying machine to lose synchronism [3]. Power System Stabilizers (PSS) are designed to suppress these oscillations and improve overall stability by applying supplementary control through the excitation controller (AVR) [4]. Conventional PSS, consisting of cascade connected lead-lag compensators derived from a linearized model of the power system around a certain operating point have long been used to damp oscillations, regardless of the varying loading conditions or disturbances.
However PSS control strategies based on linear models often fail to provide satisfactory results over a wide range of operating conditions [5].

Authors in [4, 5] presented a comprehensive approach for tuning the conventional PSS parameters and their effect on the dynamic performance of the power system. However PSS designed to damp one single oscillation mode can produce adverse effects in other modes. Several PSS design techniques have been reported $[6,7]$. Pole placement or eigenvalues methods are used in [8-11]. Classical optimization techniques failed to provide optimum PSS parameters [12]. Heuristic techniques, such as Genetic Algorithms (GA), have already been applied to PSS design [13]. A particle swarm optimization (PSO) algorithm has been used in [14], for optimizing PSS parameters. Research has been conducted in optimization using Bat algorithm in $[15,16]$. Optimization of PSS parameters, based on Bat algorithm, has been also reported [17-19]. Recently, a new synergistic control scheme, which combines control theory with heuristic optimization and computational intelligence methods, has emerged [20-22]. This study proposes an adaptive fuzzy type- 2 fast terminal synergetic power system stabilizer. Fast terminal's synergetic control parameters are determined, using the Bat optimization method, as shown in $[23,24]$. Adaptive fuzzy type-2 design is used to approximate unknown functions in the multi machine power system model.

\section{FAST TERMINAL SYNERGETIC CONTROL}

In this section, the Fast Terminal Synergetic (FTSYN) controller is developed for the following nonlinear singleinput/single-output (SISO) system:

$$
\left\{\begin{array}{l}
\dot{x}_{1}=x_{2} \\
\dot{x}_{2}=f(x, t)+g(x, t) u
\end{array}\right.
$$

where $x=\left[\begin{array}{ll}x_{1} & x_{2}\end{array}\right]^{T} \in R^{2}$ is the state vector, while $f(x, t)$ and $g(x, t)$ are unknown functions. In order to obtain the terminal convergence of the state variables, the following macrovariable is defined as a function of the state variables:

$$
\Psi=\dot{x}_{1}+\alpha x_{1}+\beta x_{1}^{\lambda}
$$


where $\alpha, \beta$ are positive constants. With a proper choice of $\lambda, \alpha$, $\beta$ and given an initial state $x_{1}(0) \neq 0$, the dynamics of the macro variable will reach the equilibrium point in a finite time. The exact time to reach zero $t_{s}$, is determined by:

$$
t_{s}=\frac{1}{\alpha(1-\lambda)} \ln \left(\frac{\alpha x_{1}(0)^{1-\lambda}+\beta}{\beta}\right) \text { (3) }
$$

and the equilibrium point at 0 is a terminal attractor. Introducing the typical constraint (4), the selected macrovariable is forced to evolve in a desired manner, despite the uncertainties and/or disturbances:

$$
T_{s} \dot{\Psi}+\Psi=0, \quad T_{s}>0
$$

where $T_{\mathrm{s}}$ is a parameter to be chosen determining the rate of convergence to the attractor and can be made arbitrary small, considering only eventual control constraint. Using (2) and (4), the macro-variable derivative is given as:

$$
\dot{x}_{2}+\alpha \dot{x}_{1}+\beta \lambda x_{2} x_{1}^{\lambda-1}=-\frac{1}{T_{s}} \Psi
$$

The fast terminal synergetic control is:

$$
u=-g^{-1}(x, t)\left(f(x, t)+\alpha x_{2}+\beta \lambda x_{2} x_{1}^{\lambda-1}+\frac{1}{T_{s}} \Psi\right)
$$

To prove the stability of fast terminal synergetic control, consider the following candidate Lyapunov function:

$$
V=\frac{1}{2} \Psi^{T} \Psi
$$

Therefore:

$$
\begin{gathered}
\dot{V}=\Psi^{T} \dot{\Psi} \\
=\Psi\left(f(x, t)+g(x, t) u+\alpha x_{2}+\beta \lambda x_{2} x_{1}^{\lambda-1}\right) \\
=-\frac{1}{T_{s}} \Psi^{2}
\end{gathered}
$$

Then:

$$
\dot{V}_{i} \leq 0
$$

\section{DESIGN OF ADAPTIVE FUZZY TYPE 2 SYNERGETIC CONTROL}

Control law (6) ensures system stabilization and robustness, but it cannot be directly implemented, since functions $f(x, t)$ and $g(x, t)$ are not known. This can be overcome by approximating functions by two interval type-2 fuzzy adaptive systems. A fuzzy system that uses type-2 fuzzy sets and/or fuzzy logic and inference, is called a type-2 fuzzy system [25]. Based on the universal approximation theorem, unknown functions $f(x, t)$ and $g(x, t)$ can be approximated by:

$$
\begin{aligned}
& \hat{f}\left(x, \theta_{f}\right)=\xi^{T}(x) \theta_{f} \\
& \hat{g}\left(x, \theta_{g}\right)=\xi^{T}(x) \theta_{g}
\end{aligned}
$$

where $\theta=\left[\theta_{1}, \theta_{2}, \ldots, \theta_{m}\right]$ is the parameters' vector, $\xi=\left[\xi_{1}, \xi_{2}, \ldots, \xi_{m}\right]^{T}$ is the vector of Fuzzy Basis Functions (FBF), such that:

$$
\begin{aligned}
& \xi^{T} \theta_{f}=\frac{1}{2}\left[\xi_{r}^{T} \xi_{l}^{T}\right]\left[\theta_{f r} \theta_{f l}\right] \\
& \xi^{T} \theta_{g}=\frac{1}{2}\left[\xi_{r}^{T} \xi_{l}^{T}\right]\left[\theta_{g r} \theta_{g l}\right]
\end{aligned}
$$

where $\xi_{l}=\left[\xi_{l}^{1}, \xi_{l}^{2}, \ldots, \xi_{l}^{m}\right]^{T}, \xi_{r}=\left[\xi_{r}^{1}, \xi_{r}^{2}, \ldots, \xi_{r}^{m}\right]^{T}$, $\theta_{r}=\left[\theta_{l r}, \theta_{2 r}, \ldots, \theta_{n v}\right]$, and $\theta_{l}=\left[\theta_{l l}, \theta_{2 l}, \ldots, \theta_{m l}\right]$.

This yields the minimum approximation error:

$$
\varepsilon=\delta_{f}+\delta_{g} u
$$

where:

$$
\begin{gathered}
\delta_{f}=f(x)-\xi^{T}(x) \theta_{f}^{*} \\
\delta_{g}=g(x)-\xi^{T}(x) \theta_{g}^{*}
\end{gathered}
$$

and $\theta_{f}^{*}, \theta_{g}^{*}$ are the optimal approximation parameters by letting:

$$
\begin{aligned}
& \tilde{\theta}_{f}=\theta_{f}-\theta_{f}^{*} \\
& \tilde{\theta}_{g}=\theta_{g}-\theta_{g}^{*}
\end{aligned}
$$

The following control law:

$$
u=-\hat{g}^{-1}(x, t)\left(\hat{f}(x, t)+\alpha x_{2}+\beta \lambda x_{2} x_{1}^{\lambda-1}+\frac{1}{T_{s}} \Psi\right)
$$

under the adaptation laws:

$$
\begin{aligned}
& \dot{\theta}_{f}=\gamma_{1} \Psi \xi(x)-\gamma_{1} \theta_{f} \\
& \dot{\theta}_{g}=\gamma_{2} \Psi \xi(x)-\gamma_{2} \theta_{g}
\end{aligned}
$$

ensures the stability of the nonlinear system (1). The Lyapunov function is chosen as:

$$
V=\frac{1}{2} \Psi^{2}+\frac{1}{2 \gamma_{1}} \widetilde{\theta}_{f}^{T} \widetilde{\theta}_{f}+\frac{1}{2 \gamma_{2}} \tilde{\theta}_{g}^{T} \tilde{\theta}_{g}
$$

Therefore :

$$
\begin{aligned}
\dot{V}= & \Psi\left(-\tilde{\theta}_{f}^{T} \xi(x)-\tilde{\theta}_{g}^{T} \xi(x) u+\varepsilon-\frac{1}{T_{s}} \Psi\right) \\
& +\frac{1}{\gamma_{1}} \tilde{\theta}_{f}^{T} \dot{\theta}_{f}+\frac{1}{\gamma_{2}} \tilde{\theta}_{g}^{T} \dot{\theta}_{g}
\end{aligned}
$$

Using (22) and (23):

$$
\dot{V}=-\frac{1}{T_{s}} \Psi^{2}-\widetilde{\theta}_{f}^{T} \dot{\theta}_{f}-\widetilde{\theta}_{g}^{T} \dot{\theta}_{g}+\varepsilon \Psi
$$

and given the following inequalities being valid:

$$
\begin{aligned}
& -\widetilde{\theta}_{f}^{T} \theta_{f} \leq-\frac{1}{2} \widetilde{\theta}_{f}^{T} \widetilde{\theta}_{f}+\frac{1}{2}\left\|\theta_{f}^{*}\right\|^{2} \\
& -\widetilde{\theta}_{g}^{T} \theta_{g} \leq-\frac{1}{2} \widetilde{\theta}_{g}^{T} \widetilde{\theta}_{g}+\frac{1}{2}\left\|\theta_{g}^{*}\right\|^{2}
\end{aligned}
$$


$\dot{V}$ can be written as:

$$
\begin{gathered}
\dot{V} \leq- \\
\frac{1}{T_{s}}\left\|\Psi^{2}+\frac{1}{2} \tilde{\theta}_{f}^{T} \tilde{\theta}_{f}+\frac{1}{2} \tilde{\theta}_{g}^{T} \tilde{\theta}_{g}+\frac{1}{2}\right\| \theta_{g}^{*} \|^{2}+|\varepsilon||\Psi|
\end{gathered}
$$

using:

$$
|\varepsilon \| \Psi|=\frac{1}{2 T_{s}}|\Psi|^{2}+\frac{T_{s}}{2}|\varepsilon|^{2}-\frac{1}{2 T_{s}}\left(|\Psi|-T_{s}|\varepsilon|\right)^{2}
$$

then:

$$
\begin{gathered}
\dot{V} \leq- \\
-\left(\frac{1}{2 T_{s}} \Psi^{2}+\frac{1}{2} \tilde{\theta}_{f}^{T} \tilde{\theta}_{f}+\frac{1}{2} \tilde{\theta}_{g}^{T} \tilde{\theta}_{g}\right)+ \\
\frac{1}{2}\left\|\theta_{f}^{*}\right\|^{2}+\frac{1}{2}\left\|\theta_{g}^{*}\right\|^{2}+\frac{T_{s}}{2}|\varepsilon|^{2}
\end{gathered}
$$

and

$$
\left\{\begin{array}{l}
\alpha=\min \left(\frac{1}{T_{s}}, \gamma_{1}, \gamma_{2}\right) \\
\mu=\frac{1}{2}\left\|\theta_{f}^{*}\right\|^{2}+\frac{1}{2}\left\|\theta_{g}^{*}\right\|^{2}
\end{array}\right.
$$

Finally:

$$
\dot{V} \leq-\alpha V+\mu+\frac{T_{s}}{2}|\varepsilon|^{2}
$$

Integrating (33) from 0 to $t$, yields:

$$
V(t) \leq-\alpha \int_{0}^{t} V(\tau) d \tau+\frac{T_{s}}{2} \int_{0}^{t}|\varepsilon(\tau)|^{2} d \tau+\mu t+V(0)
$$

Terms $\alpha \int_{0}^{t} V(\tau) d \tau$ and $\frac{T_{s}}{2} \int_{0}^{t}|\varepsilon(\tau)|^{2} d \tau$ are bounded. It can be concluded that $\Psi$ and $\dot{\Psi}$ are bounded ( $\Psi \in L_{\infty}$ and $\dot{\Psi} \in L_{\infty}$ ). Since $\varepsilon, \widetilde{\theta}_{f}$ and $\widetilde{\theta}_{g}$ are bounded, hence $V(t)$ is also bounded, guaranteeing the stability of the closed loop system.

To optimize the synergetic parameters $T_{s}, \alpha, \beta$ and $\lambda$, a fuzzy synergetic approach using Bat algorithm is employed. The typical values of the optimized parameters are taken as $[0.05-0.2]$ for $T_{s},[100-300]$ for $\alpha,[50-150]$ for $\beta$ and $[1-10]$ for $\lambda$.

\section{BAT OPTIMIZATION ALGORITHM}

Bat algorithm is a relatively new meta-heuristic optimization method [23]. This algorithm exploits the so-called echolocation of bats. Bats use sonar echoes to detect and avoid obstacles. They navigate by emitting high frequency sounds waves and detecting the time delay of the reflected waves. From the detected time delay, bats know how far away they are from the prey or the obstacle [25].

The algorithm, with the use of random walks (one solution is selected among the current best solutions and then the random walk is applied to generate a new solution for each bat), is presented in detail in [23, 24]. Applying the Bat optimization algorithm and after the optimization procedure, we find $T_{s}=0.1000, \alpha=240, \beta=140$ and $\lambda=3$.

\section{POWER SYSTEM MODEL}

The nonlinear power system model considered in this paper represents a synchronous machine connected to an infinite bus via a double circuit transmission line. A nonlinear representation of the power system, considered during a transient period after a major disturbance has occurred, is given by $[18,19]$ :

$$
\left\{\begin{array}{l}
\Delta \dot{\omega}=\Delta P / M \\
\Delta \dot{P} / M=f(x)+g(x) u
\end{array}\right.
$$

where $\Delta \omega$ is the speed deviation, $\Delta P=P_{m}-P_{e}$ the accelerating power, $M$ is the inertia coefficient, $u \in R$ is the input, $f(x)$ and $g(x)$ are nonlinear functions and $g(x) \neq 0$ in the controllability region.

The block diagram of a conventional lead-lag power system stabilizer is shown in Figure 1. $T_{w}$ is the wash out time constant, $T_{1 i}-T_{4 i}$ are the PSS time constants and $K_{i}$ is the PSS parameter of generator $i$. The optimal parameters of the conventional PSS are obtained by BAT method and are listed in Table II [19].

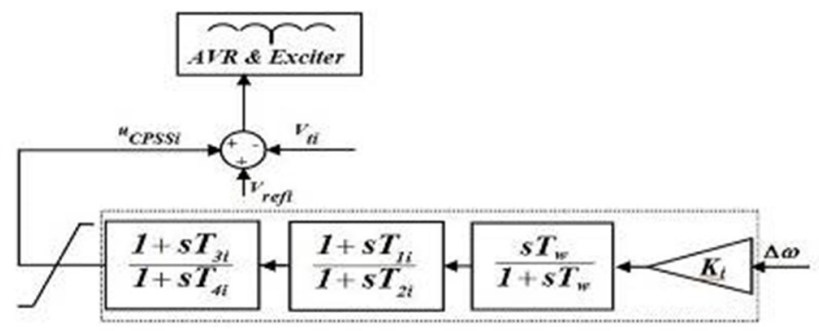

Fig. 1. Conventional power system stabilizer

\section{SimUlation RESULTS}

To proof the robustness and effectiveness of the proposed optimal fuzzy synergetic PSS, simulations were carried out under different operating conditions of the multi-machine power system. To demonstrate the stability enhancement achieved with the proposed stabilizer, a three-phase fault test is applied at bus 7 of the multi-machine power system, with duration of $60 \mathrm{~ms}$ before its clearance. Seven fuzzy sets were used for each variable of the proposed PSS. The fuzzy sets for $\Delta P \in[-2.5730,0.55]$ and $Q \in[-0.1086,1.8143]$ are defined according to the membership functions shown in Figures 2 and 3 respectively.

Three disturbance scenarios have been considered in the simulation, in order to test the robustness of the proposed control scheme. Table I describes these three cases. In each case, the proposed stabilizer is compared with a BAT CPSS and an AFT2 SYNPSS. 
TABLE I. CASES OF LOADING CONDITIONS FOR THE SYSTEM (PU)

\begin{tabular}{|c|c|c|c|c|}
\hline & & \multicolumn{3}{|c|}{ Generator } \\
\hline & & G1 & G2 & G3 \\
\hline \multirow{2}{*}{ Light case } & P & 0.9649 & 1.00 & 0.45 \\
\cline { 2 - 5 } & $\mathbf{Q}$ & 0.223 & -0.1933 & -0.2668 \\
\hline \multirow{2}{*}{ Normal case } & $\mathbf{P}$ & 1.7164 & 1.630 & 0.85 \\
\cline { 2 - 5 } & $\mathbf{Q}$ & 0.6205 & 0.0665 & -0.1086 \\
\hline \multirow{2}{*}{ Heavy case } & $\mathbf{P}$ & 3.5730 & 2.20 & 1.35 \\
\cline { 2 - 5 } & $\mathbf{Q}$ & 1.8143 & 0.7127 & 0.4313 \\
\hline
\end{tabular}

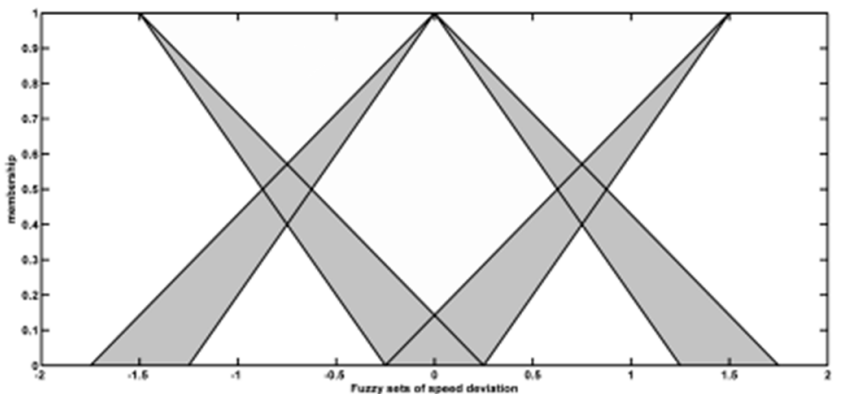

Fig. 2. Fuzzy sets for speed deviation

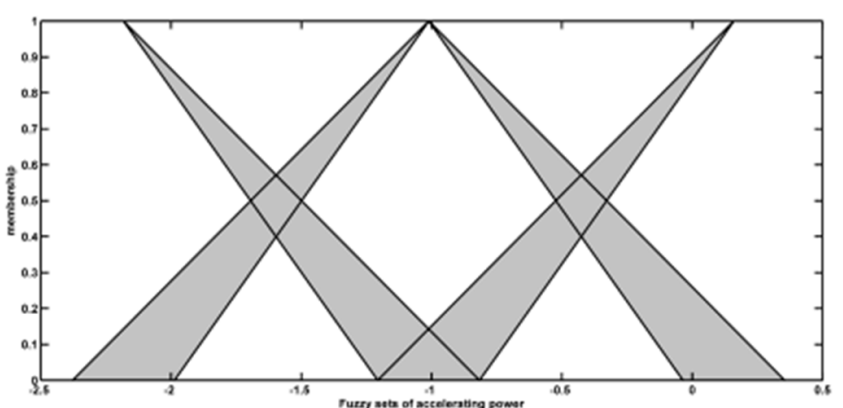

Fig. 3. Fuzzy sets for accelerating power

TABLE II. BAT CONVENTIONAL POWER SYSTEM STABILIZER PARAMETETERS

\begin{tabular}{|l|c|c|c|}
\hline & K & T1 & T3 \\
\hline BATPSS1 & 46.6588 & 0.4153 & 0.2698 \\
\hline BATPSS2 & 8.4751 & 0.4756 & 0.1642 \\
\hline BATPSS3 & 4.2331 & 0.2513 & 0.1853 \\
\hline
\end{tabular}

The three-machine test system, used to examine the interarea oscillation control problem, is shown in Figure 4 for light load case, in Figure 5 for nominal load case and in Figure 6 for a heavy load case.

(a)

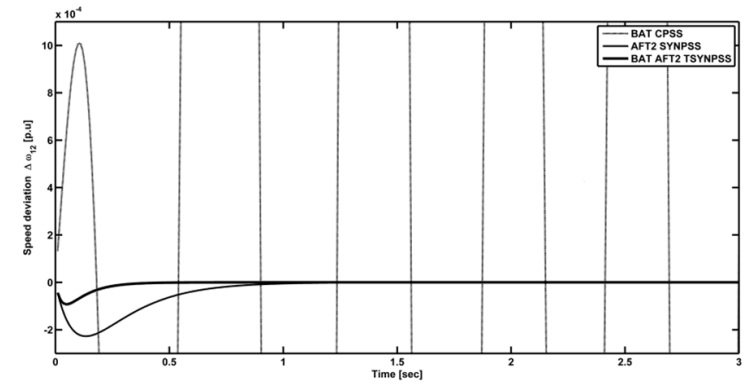

(b)

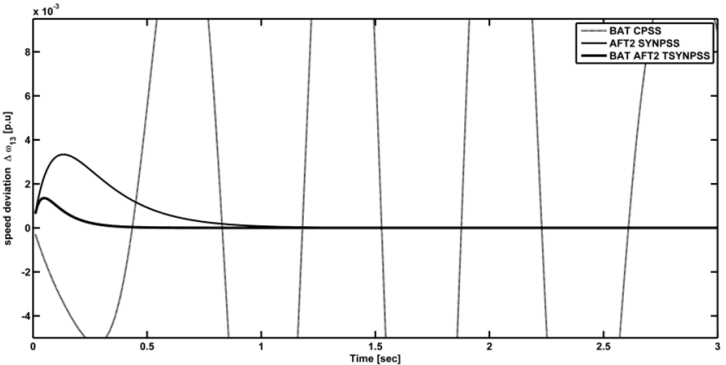

(c)

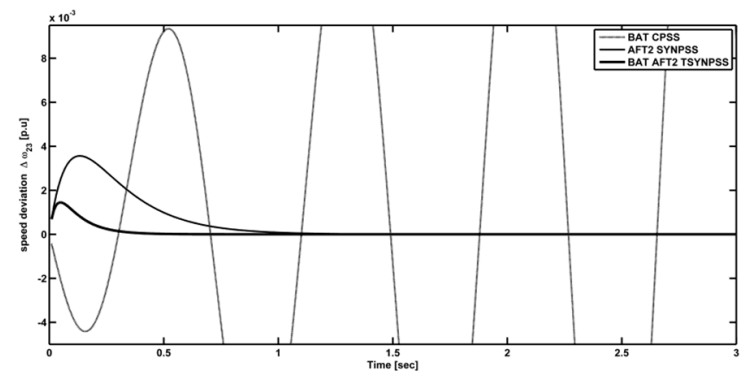

Fig. 4. Light load scenario

(a)

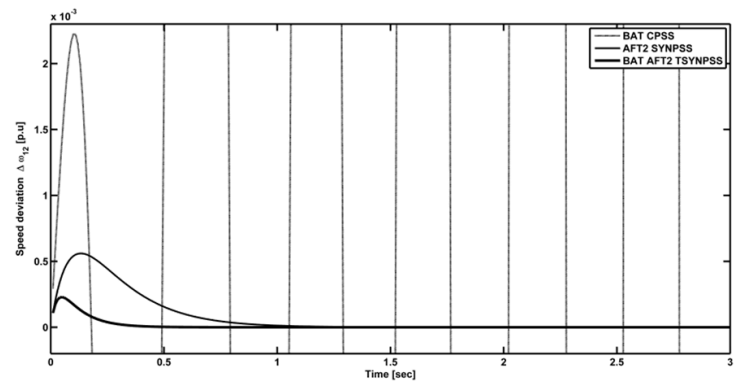

(b)

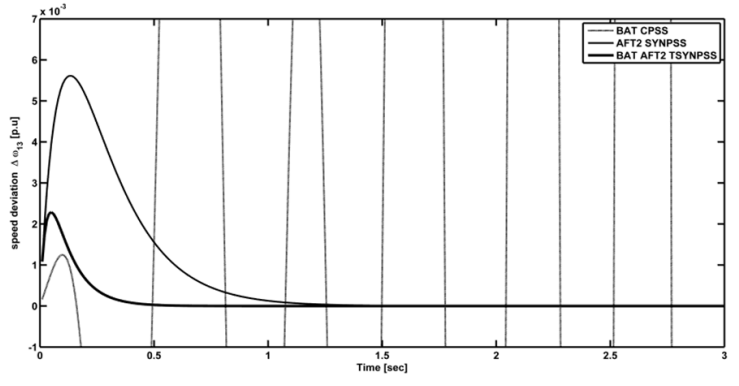

(c)

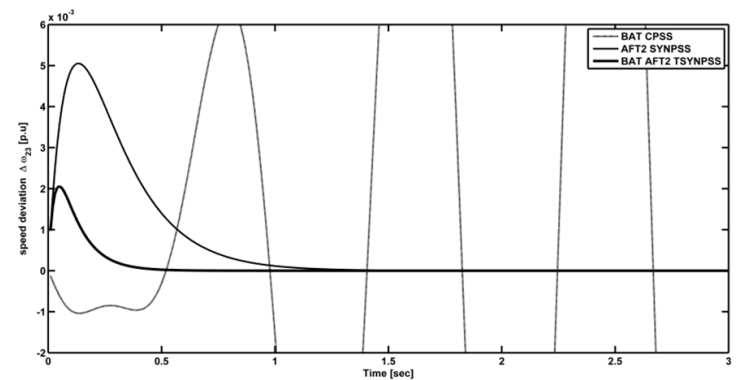

Fig. 5. Normal load scenario 
(a)

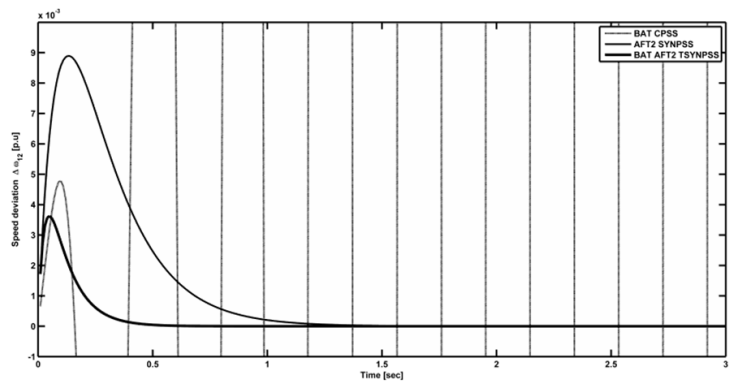

(b)

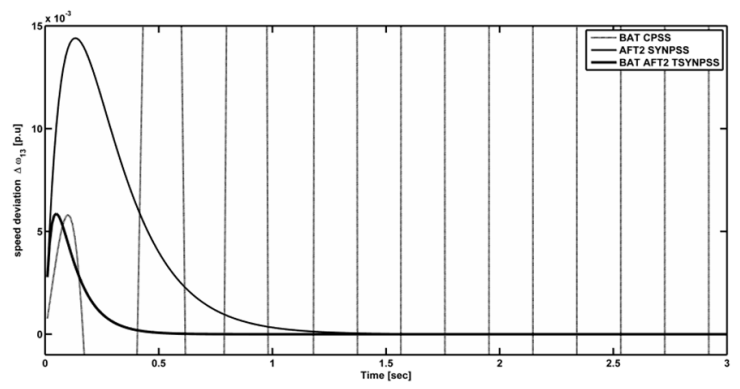

(c)

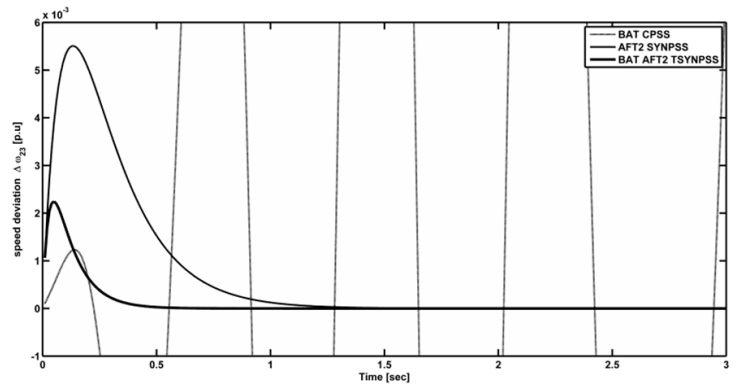

Fig. 6. Heavy load scenario

The proposed stabilizer keeps the generator synchronized. Three studies have been performed to investigate the effect of the proposed BAT AFT2 SYNPSS. The results are compared with a BAT CPSS and an AFT2 SYNPSS. The fault is cleared and the proposed stabilizer helps the system to reach a stable operating point very quickly.

\section{CONCLUSION}

In this paper a BAT algorithm was used in combination with an adaptive fuzzy type-2 terminal synergetic (BAT AFT2) to design a PSS for a multi machine power system. The obtained results show that the proposed stabilizer is very effective and can cope with different loading conditions. The proposed stabilizer could rapidly damp oscillations that would eventually lead to a loss of synchronism. The simulation results demonstrate the superior performance of the proposed PSS over the BAT conventional power system stabilizer (BAT CPSS) and adaptive fuzzy type-2 synergetic power system stabilizer (AFT2 SYNPSS).

\section{REFERENCES}

[1] F. P. Demello, "Concepts of synchronous machine stability as affected by excitation control", IEEE Transactions on Power Appparatus and Systems, Vol. 88, No. 4, pp. 316-329, 1969
[2] K. Tang, G. K. Venayagamoorthy, "Damping inter-area oscillations using virtual generator based power system stabilizer", Electric Power Systems Research, Vol. 129, pp. 126-141, 2015

[3] E. V. Larsen, D. A. Swann, "Applying power system stabilizers part II: Performance objectives and tuning concepts", IEEE Transactions on Power Apparatus and Systems, Vol. 100, No. 6, pp. 3025-3033, 1981

[4] P. Kundur, N. J. Balu, M. G. Lauby, Power System Stability and Control, McGraw-Hill, 1994

[5] P. M. Anderson, A. A. Fouad, H. Happ, Power System Control and Stability, IEEE, 1979

[6] A. Ghosh, G. Ledwich, O. P. Malik, G. S. Hope, "Power system stabilizer based on adaptive control techniques", IEEE Transactions on Power Apparatus and Systems, Vol. 103, No. 8, pp. 1983-1989, 1984

[7] S. J. Cheng, Y. S. Chow, O. P. Malik, G. S. Hope, "An adaptive synchronous machine stabilizer", IEEE Transactions on Power Systems, Vol. 1, No. 3, pp. 101-107, 1986

[8] H. M. Soliman, H. A. Yousef, "Saturated robust power system stabilizers", International Journal of Electrical Power \& Energy Systems, Vol. 73, pp. 608-614, 2015

[9] A. Y. Sivaramakrishnan, M. V. Hariharan, M. C. Srisailam, "Design of variable-structure load-frequency controller using pole assignment technique", International Journal of Control, Vol. 40, No. 3, pp. 487 498, 1984

[10] M. L. Kothari, J. Nanda, K. Bhattacharya, "Design of variable structure power system stabilisers with desired eigenvalues in the sliding mode", IEE Proceedings C-Generation, Transmission and Distribution, Vol. 140, No. 4, pp. 263-268, 1993

[11] K. Bhattacharya, M. L. Kothari, J. Nanda, "Design of discrete-mode variable structure power system stabilizers", International Journal of Electrical Power \& Energy Systems, Vol. 17, No. 6, pp. 399-406, 1995

[12] J. Talaq, "Optimal power system stabilizers for multi machine systems", International Journal of Electrical Power \& Energy Systems, Vol. 43, No. 1, pp. 793-803, 2012

[13] F. M. Adjeroud, F. Djahli, A. Mayouf, T. Devers, "A coordinated genetic based type-2 fuzzy stabilizer for conventional and superconducting generators", Electric Power Systems Research, Vol. 129 , pp. 51-61, 2015

[14] M. S. Mahmoud, H. M. Soliman, "Design of robust power system stabilizer based on particle swarm optimization", Circuits and Systems, Vol. 3, No. 1, pp. 82-89, 2012

[15] A. S. Oshaba, E. S. Ali, S. A. Elazim, "MPPT control design of PV system supplied SRM using BAT search algorithm", Sustainable Energy, Grids and Networks, Vol. 2, pp. 51-60, 2015

[16] A. H. Gandomi, X. S. Yang, "Chaotic bat algorithm", Journal of Computational Science, Vol. 5, No. 2, pp. 224-232, 2014

[17] M. R. Sathya, M. M. T. Ansari, "Load frequency control using bat inspired algorithm based dual mode gain scheduling of PI controllers for interconnected power system", International Journal of Electrical Power \& Energy Systems, Vol. 64, pp. 365-374, 2015

[18] D. K. Sambariya, R. Prasad, "Robust tuning of power system stabilizer for small signal stability enhancement using metaheuristic bat algorithm", International Journal of Electrical Power \& Energy Systems, Vol. 61, pp. 229-238, 2014

[19] E. S. Ali, "Optimization of power system stabilizers using BAT search algorithm", International Journal of Electrical Power \& Energy Systems, Vol. 61, pp. 683-690, 2014

[20] A. Kolesnikov, G. Veselov, and A. Kolesnikov, "Modern applied control theory: synergetic approach in control theory," TRTU, Moscow, Taganrog, Russia, pp. 4477-4479, 2000

[21] A. Kolesnikov, G. Veselov, A. Monti, F. Ponci, E. Santi, R. Dougal, "Synergetic synthesis of DC-DC boost converter controllers: $\tau$ heory and experimental analysis", Seventeenth Annual IEEE Applied Power Electronics Conference and Exposition, Dallas, USA, March 10-14, 2002

[22] Z. Jiang, "Design of a nonlinear power system stabilizer using synergetic control theory", Electric Power Systems Research, Vol. 79, No. 6, pp. $855-862,2009$ 
[23] X. S. Yang, “A new metaheuristic bat-inspired algorithm”, Nature Inspired Cooperative Strategies for Optimization, pp. 65-74, 2010

[24] X. S. Yang, X. He, "Bat algorithm: literature review and applications", International Journal of Bio-Inspired Computation, Vol. 5, No. 3, pp. 141-149, 2013

[25] E. Nechadi, M. N. Harmas, A. Hamzaoui, N. Essounbouli, "Type-2 fuzzy based adaptive synergetic power system control", Electric Power Systems Research, Vol. 88, pp. 9-15, 2012 